\title{
The Properties of Relative Regularity and Compactness
}

\author{
Genglei Li \\ College of Science, Tianjin Polytechnic University \\ Tianjin , 300160, China \\ E-mail:lglxt@126.com \\ Huidong $\mathrm{Wu}$ \\ College of Business, Hebei Normal University \\ Shijiazhuang, 050000, China \\ E-mail:xtlqjz@sohu.com
}

\begin{abstract}
In this paper, some topological properties were studied, especially including relative Hausdorff, relative regular and relative strongly regular and the property of nearly-paracompact under the perfect mapping was also discussed .
\end{abstract}

Keywords: $\mathrm{Y}$ is Hausdorff in $\mathrm{X}, \mathrm{Y}$ is regular in $\mathrm{X}, \mathrm{Y}$ is strongly regular in $\mathrm{X}, \mathrm{Y}$ is nearly-paracompact in $\mathrm{X}$.

\section{Introduction}

Relative topological properties are extension of classic topological invariants.In 1989, the relative topological properties were discussed by A.V.Archangel'skii and H.M.M.Genecli in Note 1, and A.V.Arhangel'skii gave the first systematic text on relative topological properties in 1996. In recent years, some further new results of the relative topology were obtained respective by A.V.Arhangel'skii, J.Tartir and W.Just, O.Pavlov and M.Matveer, I.Yaschenko, V.V.Tkachuk, M.G.Tkachenko and R.G.Wilson, etc.

In my paper, some relative topological properties were studied and some results were given.

\section{The Properties of Relative Regularity}

$\mathrm{X}$ is a space, $\mathrm{Y} \subset \mathrm{X}$, the concept of $\mathrm{Y}$ is Hausdorff, regular, superregular and strongly regular in $\mathrm{X}$ were introduced in Note 1. In this part, some properties of them were discussed, and I gave there results.

Definition 2.1 $\mathrm{Y}$ is Hausdorff in $\mathrm{X}$ : If for every two distinct points $\mathrm{x}$ and $\mathrm{y}$ of $\mathrm{Y}$, there are disjoint open subsets $\mathrm{u}$ and $\mathrm{v}$ of $X$, such that: $\mathrm{x} \in \mathrm{u}$ and $\mathrm{y} \in \mathrm{v}$.

Definition 2.2 $\mathrm{Y}$ is regular in $\mathrm{X}$ : If for each $\mathrm{y}$ of $\mathrm{Y}$ and each closed subset $\mathrm{p}$ of $\mathrm{X}$, such that $\mathrm{y} \notin \mathrm{p}$, there are disjoint open subsets $\mathrm{u}$ and $\mathrm{v}$ of $\mathrm{X}$, such that: $\mathrm{y} \in \mathrm{u}$ and $\mathrm{p} p \cap Y \subset \mathrm{v}$.

Definition 2.3 $\mathrm{Y}$ is superregular in $\mathrm{X}$ : If for each $\mathrm{y}$ of $\mathrm{Y}$ and each closed subset $\mathrm{p}$ of $\mathrm{X}$, such that $\mathrm{y} \notin \mathrm{p}$, there are disjoint open subsets $\mathrm{u}$ and $\mathrm{v}$ of $\mathrm{X}$, such that: $\mathrm{y} \in \mathrm{u}$ and $p \subset v$.

Definition 2.4 $\mathrm{Y}$ is strongly regular in $\mathrm{X}$ : If for each $\mathrm{x}$ of $\mathrm{X}$ and each closed subset $\mathrm{p}$ of $\mathrm{X}$, such that $\mathrm{y} \notin \mathrm{p}$, there are disjoint open subsets $\mathrm{u}$ and $\mathrm{v}$ of $\mathrm{X}$, such that: $\mathrm{x} \in \mathrm{u}$ and $p \cap Y \subset \mathrm{v}$.

Theory 2.5 If $\mathrm{Y}$ is a dense subspace of a space $\mathrm{X}$. Then, $\mathrm{Y}$ is Hausdorff in $\mathrm{X}$ if and only if $\mathrm{Y}$ is Hausdorff.

Proof. “ $\Rightarrow$ " Let $y_{1}, y_{2}$ are arbitrary points of $\mathrm{Y}$. Since $\mathrm{Y}$ is Hausdorff in $\mathrm{X}$, so there exist two disjoint open subsets $u_{1}$ and $v_{1}$ in $\mathrm{X}$, such that: $y_{1} \in u_{1}$ and $y_{2} \in v_{1}$. We may assume that: $u=u_{1} \cap Y$ and $v=v_{1} \cap Y$. Then, $\mathrm{u}$ and $\mathrm{v}$ are two open sets in $\mathrm{Y}$ and such that: $u \cap v=\varnothing, y_{1} \in u$ and $y_{2} \in v$. That is $\mathrm{Y}$ is Hausdorff.

" $\Leftarrow$ " Let $y_{1}, y_{2}$ are arbitrary points of $\mathrm{Y}$. Since $\mathrm{Y}$ is Hausdorff, so there exist two disjoint open subsets $u_{1}$ and $v_{1}$ in $\mathrm{Y}$, such that: $y_{1} \in u_{1}$ and $y_{2} \in v_{1}$. So there exist two open sets $\mathrm{u}$ and $\mathrm{v}$ in $\mathrm{X}$, such that: $u_{1}=u \cap Y, v_{1}=v \cap Y$. As follow we will prove $u \cap v=\varnothing$. We may also assume that $u \cap v \neq \varnothing$. Then $u \cap v$ is open in $\mathrm{X}$ since $\mathrm{u}$ and $\mathrm{v}$ are 
open in $\mathrm{X}$, and $\mathrm{Y}$ is a dense subspace of $\mathrm{X}$, so $(u \cap v) \cap Y \neq \varnothing$, therefore $u_{1} \cap v_{1} \neq \varnothing$. This is contradict with $u_{1} \cap v_{1}=\varnothing$. So, $u \cap v=\varnothing$. That is $\mathrm{Y}$ is Hausdorff in $\mathrm{X}$.

Theory 2.6 If $\mathrm{Y}$ is closed-open subspace of $\mathrm{X}$. Then, $\mathrm{Y}$ is regular in $\mathrm{X}$ if and only if $\mathrm{Y}$ is superregular in $\mathrm{X}$.

Proof. " $\Rightarrow$ Let $\mathrm{y}$ is an arbitrary point of $\mathrm{Y}$ and an arbitrary closed subset $\mathrm{p}$ of $\mathrm{X}$, such that $\mathrm{y} \notin \mathrm{p}, \quad$ since $\mathrm{Y}$ is regular in $\mathrm{X}$, there are disjoint open subsets $u_{1}$ and $v_{1}$ of $\mathrm{X}$, such that: $\mathrm{y} \in u_{1}$ and $p \cap Y \subset v_{1}$. We may assume that $u=Y \cap u_{1}$, then Since $\mathrm{y}$ is a point of $\mathrm{Y}$ and $\mathrm{Y}$ is open in $\mathrm{X}$, so $\mathrm{u}$ is an open set of $\mathrm{X}$, and such that: $y \in u$. Since $\mathrm{Y}$ is closed in $\mathrm{X}$, so $X / Y$ is an open set of $\mathrm{X}$. We may also assume that: $v=v_{1} \cup(X / Y)$, then we can get: $p \subset v$ and $u \cap v=\varnothing$. That is $\mathrm{Y}$ is superregular in $\mathrm{X}$.

" $\Leftarrow$ " Let $\mathrm{y}$ is an arbitrary point of $\mathrm{Y}$ and an arbitrary closed subset $\mathrm{p}$ of $\mathrm{X}$, such that $\mathrm{y} \notin \mathrm{p}$, there are disjoint open subsets $u$ and $v$ of X, such that: $y \in u$ and $p \subset v$. Obviously, $p \cap Y \subset v$. That is $\mathrm{Y}$ is regular in $\mathrm{X}$.

Theory $2.7 \mathrm{Y}$ is strongly regular in $\mathrm{X}$ if and only if for each point $\mathrm{x}$ of $\mathrm{X}$ and arbitrary open set $u$ of $\mathrm{X}$, such that $x \in X$, there exist an open $u_{x}$ of $\mathrm{X}$, such that $\mathrm{y} x \in u_{x} \subset u$ and $\overline{u_{x}} \cap(Y \backslash u)=\varnothing$.

Proof. " $\Rightarrow$ Let $\mathrm{x}$ is an arbitrary point of $\mathrm{Y}$ and an arbitrary open subset $\mathrm{u}$ of $\mathrm{X}$, such that $\mathrm{x} \in \mathrm{p}$. Let $\mathrm{p}=\mathrm{X} \backslash \mathrm{u}$, then $\mathrm{p}$ is closed in $\mathrm{X}$ and $y \notin p$. Since $\mathrm{Y}$ is strongly regular in $\mathrm{X}$, so there are disjoint open subsets $u_{1}$ and $v_{1}$ of $\mathrm{X}$, such that: $\mathrm{x} \in u_{1}$ and $p \cap Y=Y \backslash u \subset v_{1}$. We may let $u_{x}=u_{1} \cap u$, therefore, $x \in u_{x} \subset u$ and $\overline{u_{x}} \cap(Y \backslash u)=\varnothing$.

" $\Leftarrow$ " Let $\mathrm{x}$ is an arbitrary point of $\mathrm{X}$ and $\mathrm{p}$ is an arbitrary closed subset of $\mathrm{X}$, such that $\mathrm{x} \notin \mathrm{p}$. Let $\mathrm{u}=\mathrm{X} \backslash \mathrm{p}$. Then $x \in u$. So there is an open set $u_{x}$ of $\mathrm{X}$, such that: $x \in u_{x} \subset u$ and $\overline{u_{x}} \cap(Y \backslash u)=\varnothing$. Since $\mathrm{Y} \backslash \mathrm{u}=p \cap Y$, So we can assume: $u_{1}=u_{x}$ and $u_{2}=X \backslash \bar{u}_{x}$. Obviously, $x \in u_{1}, p \cap Y \subset u_{2}$ and $u_{1} \cap u_{2}=\varnothing$. That is $\mathrm{Y}$ is strongly regular in $\mathrm{X}$.

\section{The Property of Relative Compactness under the Perfect Mapping.}

The definition of nearly-paracompact was introduced in Note 3. Some properties of topological spaces under the perfect mapping were given in Note 6 . In this part, I studied the property of nearly-paracompact under the perfect mapping, and gave a result about it.

Definition $3.1 \mathrm{Y}$ is nearly-paracompact in $\mathrm{X}$ : If for each open covering $\mathrm{A}$ of $\mathrm{X}$, there exists an open family covering $\mathfrak{R}$ of $\mathrm{Y}$, such that: $\mathfrak{R}$ refines $\mathrm{A}$, and $\mathfrak{R}$ is locally finite at each $\mathrm{y}$ of $\mathrm{Y}$.

Theorem 3.2 Let $\mathrm{f}: \mathrm{X} \rightarrow \mathrm{Y}$ is a perfect mapping. If $Y_{1}$ is nearly-paracompact in Y.Then $f^{-1}\left(Y_{1}\right)$ is nearly-paracompact in $\mathrm{X}$.

Proof. Let $\mathrm{A}=\left\{u_{s}: s \in S\right\}$ is an open covering of $\mathrm{X}$. Since $\mathrm{f}$ is a perfect mapping, so for each $\mathrm{y} \in \mathrm{Y}$, the fiber $f^{-1}(y)$ is a compact subset of $\mathrm{X}$. Thus, there exists a finite subset $s(y)$ of $\mathrm{S}$, such that: $f^{-1}(y) \subset \bigcup_{s \in S(y)} u_{s}=u_{y(s)}$. Since $\mathrm{f}$ is a perfect mapping, by the TH1.4.13 in Note 6, there exists an open neighborhood $w_{y(s)}$ of $\mathrm{y}$, such that $: f^{-1}(y) \subset f^{-1}\left(w_{y(s)}\right) \subset u_{y(s)}$. We may also assume that: $f^{-1}\left(w_{y(s)}\right)$ is $v_{y(s)}$. That is $v_{y(s)}=f^{-1}\left(w_{y(s)}\right)$. Then, it is obvious that: $v_{y(s)}$ is open in $\mathrm{X}$ and such that: $f^{-1}(y) \subset v_{y(s)}=f^{-1}\left(f\left(v_{y(s)}\right)\right) \subset u_{y(s)}$ and $f\left(v_{y(s)}\right)$ is an open subset of $\mathrm{Y}$. Obviously, $\mathfrak{R}_{1}=\left\{f\left(v_{y(s)}\right): y \in Y\right\}$ is an open covering of $\mathrm{Y}$. Since $Y_{1}$ is nearly-paracompact in $\mathrm{Y}$, so there exists an open family covering $\mathfrak{R}_{2}=\left\{v_{a}: a \in A\right\}$ of $\mathrm{Y}$ by open subsets of $Y_{1}$, such that: $\mathfrak{R}_{2}$ refines $\mathfrak{R}_{1}$, and $\mathfrak{R}_{2}$ is locally finite at each $\mathrm{y} \in \mathrm{Y}$. We may also assume that $f\left(v_{y(s)}\right)$ which contains $v_{a}$ is $f\left(y_{a}(s)\right)$. Since $\mathrm{f}$ is perfect mapping ,thus, $\mathfrak{R}_{3}=\left\{f^{-1}\left(v_{a}\right) ; a \in A\right\}$ is an open family of $\mathrm{X}$ and locally Finite each $x \in f^{-1}\left(Y_{1}\right)$. Obviously, $f^{-1}\left(v_{a}\right) \subset f^{-1}\left(f\left(v_{y(s)}\right)\right) \quad=v_{y_{a}(s)}=u_{y_{a}(s)}$. Let $\mathfrak{R}=\left\{f^{-1}\left(v_{a}\right) \cap u_{s} \cap Y: a \in A, s \in S_{a}(y)\right\}$. Then , $\mathfrak{R}$ is an open family covering of $\mathrm{Y}$ and such that $\mathfrak{R}$ refines $\mathrm{A}$, and $\mathfrak{R}$ is locally finite at each $\mathrm{x} \in f^{-1}\left(Y_{1}\right)$. That is $f^{-1}\left(Y_{S}\right)$ is nearly-paracompact in $\mathrm{X}$. 


\section{References}

A.V.Arhangel'skii. (1996). Relative topological properties and relative topological spaces. Topology and Appl.20, 1-13.

A.V.Arhangel'skii and T.Nogura. (1998). Relative sequentiality. Topology and Appl. 82, 49-58.

A.V.Arhangrl'skii.(2002). From classic topological invariants to relative topological properties. Scientiae mathematicae japonicae,55, No. 1 153-201.

A.V.Arhangel'skiiandI.Ju.Gordienko.(1994). Locally Finite Topological Spaces. Questions and Answers in General Topology. 12:1.

Baturov D.P.(1990). Normality in dense subspaces of products. Topology and Appl.36, 111-116.

R.Engelking.(1997). General topology. Warszawa.(Chapter 1 ). 